\title{
A Study on Traditional Decorative Water Patterns
}

\author{
Xie $\mathrm{Mei}^{1, \mathrm{a}}$ \\ ${ }^{1}$ College of Arts, Taiyuan University of Technology, Jinzhong, Shanxi, China \\ a261427893@qq.com
}

Keywords: water pattern, cultural connotation, changes.

\begin{abstract}
Water pattern, one of Chinese traditional decorative patterns, has rich humanistic background. It was produced in the Neolithic Age, and still in use up to now. Since water patterns image has been constantly inherited and innovated in history, this paper tried to present its features in different times based on the production background and social changes.
\end{abstract}

\section{Humanistic background of water patterns image}

Since ancient times, human's dependence on water has been always superior to the need of other material resources for survival. There were certain reasons why primitive ancients chose to live in river basin with abundant water in agricultural society. With the gradual development of productivity, the realization of water as necessity and source of life has been increasingly deepened by primitive ancients. Due to the dependence on water which is essential, primitive ancients made their life defined near rivers in the case of drawing water easily. Xu Xusheng in his book Legendary Period in Ancient China wrote that primitive ancients could estimate the changes of water level in different seasons based on their life experiences. They define the highest water level when the tide rises as the boundary and settle the whole clan down near the boundary. Possibly it was concerned with the reason why the world civilizations were all produced around rivers. It was shown that primitive ancients could be completely able to realize the independent relationship between their living and production and water. Moreover, water was the most basic material resource for survival in primitive society. But it was also something both favorable and daunting for primitive ancients. It was not only the source of life but also the staggering damage. On the basis of such long-time precipitation of mental experience, primitive ancients came to have a specific feeling: the consciousness of worship for water. The consciousness was expressed in numerous records of ancient books, philosophical thoughts, worship for spring and sea and many decorative arts.

This kind of consciousness has gradually infiltrated into human thoughts. It was realized that the lifeblood of agricultural society was exactly water. Moreover, "in the perspective of a murmuring stream, surging water and roaring river, the rich farming awareness and warm national instincts of primitive ancients can easily make themselves introspect. And then, they could produce the self-awareness from observing things to reviewing themselves. Thus, it became especially rich in philosophy, emotion and virtue for human's understanding and elucidation of water." And also, such kind of nature made ancient people start to summarize the moral character of water from the beginning observation of its natural state and then expound to reflect on life, society and the universe. Consequently, although water was just a common substance, it has been an important concept in Chinese traditional culture. In the theory of the five elements (held by the ancients to compose the physical universe and later used in traditional Chinese medicine to explain various physiological and pathological phenomena), "water, fire, wood, metal and earth" symbolized all of nature and "water" was put at first. In Taiyi Sheng Shui, the idea was put forward that Taiyi (also known as doctrines of Taoism) produced water and it complemented Taiyi in return, and as a result nature was produced; and then nature complemented Taiyi, and the world came into being. It was also emphasized in this book that water was produced by Taiyi, and was the source of all things on earth. It can be seen that ancient people ranked water very highly, which was reflected in many naive philosophy systems in ancient China. For instances: in Confucius, water was compared to virtue; in Taoism, water was likened to doctrine; in Buddhist, water was used to view Buddha. Various interpretations made water 
have increasingly abundant connotations. Besides, water was also repeatedly chanted as an aesthetic image in literary more than a philosophy prototype in philosophical system. For example, Nineteen Ancient Poems has described that "The girl and her lover, knowing they'll never join together, are in silence watching other." In The Reed, the poet wrote that "Green, green the reed, Dew and frost gleam. Where's she I need? Beyond the stream." In the poem A Greeting on the Huai River to My Old Friends from Liangchuan by Wei Yingwu, he wrote that "Since we left one another, floating apart like clouds. Ten years have run like water-till at last we join again." Also in Li Bai's Our Parting at Kinling Inn, the poet wrote that "Now prithee ask the River that ever eastward flows, if any parting constant as his he ever knows?" The numerous literary works used water to be compared to images, like woman, time and emotion. They all took advantage of water as the specific representative to express emotion.

Such kind of water-based thought has rooted in Chinese traditional culture through the inheritance from ancient times. It also has influenced on the creation of decorative patterns as traditional culture. This complex emotion towards water was presented earliest in water patterns with mystery characteristics.

The primitive thought in the embryonic stage has been affected by sorcery activity and primitive religion faith for a fairly long time. It could not entirely create decorative patterns from aesthetic angle as well could not reappear aesthetic pursuit wholly. The painting of patterns, to a great extent, was related to totem and sorcery, which resulted in the mysterious temperament of primitive patterns. It could date back to the rotation pattern of painted pottery about the mysterious water patterns. There were generally Shaman coma phantom and biography written transfer mode etc. as regards the origins of the rotation pattern of painted pottery. In this paper, the sketching of water was applied as the origin since the generation of a pattern should come from aesthetic experiences of people during living and production. In fishing-and-hunting times, the intimate contact with water stimulated human's creation on aesthetic practice. The eddy in swift current and the ripples formed when net casting, rock falling and fish swimming were both main rhythms with which people can contact during daily fishing and hunting. Through such production practice, the eddy water pattern could come into being. Moreover, the opinion that the production of swirling grain derived from water was more constant than other opinions about origins.

\section{The development of water patterns in different historical periods}

There were various kinds of guesses about the origin of water pattern. Therefore, many types of image were also put forward to present water pattern. In this paper, water pattern was divided into two forms as romantic and realistic before and after Wei and Jin dynasties respectively. On one hand, when we made a comparison of the water pattern before and after Wei and Jin dynasties, it can be found that plenty of imagination was shown in early dynasties, and that abstract lines were used to express vivid water images. Therefore, I referred the early water pattern to be "mysteriously romantic" in this article. On the other hand, the water pattern has gradually been much real and more meticulous in late dynasties. It was referred to be "realistically romantic".

Two conditions should be mainly considered when dividing water patterns: first, the lines of water pattern; second, the modeling of water pattern. Before Wei-and-Jin periods, water patterns were mainly swirling grain and rippling grain. The two grain types were not only completely abstract but also various and vivid in these periods. And in the Neolithic Age, lines-based water patterns were expressed majorly as spiral lines of painted pottery in Majia Kiln. The swirling grain was a simply static presentation, and it could form dynamic effects through the combination of outer shape and inner power at this time. Moreover, in this period, the mysterious romanticism of decorative patterns was chiefly presented through spinning rhythm formed by rich spiral curve and dense curves. The basic element regarded the spiral concentric circles which were spread around from one centre and S-shaped or C-shaped curves as the primary pattern. And people at this time could freely organize these basic elements to form rhythms with flowing curve by employing the similar decorative patterns as fit pattern by itself, two sides continual image and four sides continual image. Since the period from original decorative patterns of painted pottery to Shang-and-Zhou dynasties, there were 
not exact patterns to express "water". But due to the continuity of form, cloud thunder pattern and revolving pattern involved were both attributed into water patterns. And except the tendency to inherit swirling grain in form expression, the ancient man had also a clear understanding of water changes and law of motion when observing the weather change law in nature. They mastered such a kind of change rule that water generated gas and then cloud was produced accordingly. And they also know the fact that the process could be established reversely. As a result, I referred cloud thunder pattern at this time as the gasification of water or the apotheosis of water from the angle of social environment that the religious authority was supreme.

In Shang-Zhou dynasties, the cloud thunder patterns have succeeded to the basic forms of swirling pattern. It centered on e and square spiraling grain as basic element, and formed two sides continual image based on S-shape and C-shape. Cloud thunder pattern usually spread on bronze wares in a complicated and overlapped integral shape without the slippery spiral and orderly arrangement, which was full of ritual severity and theocratic awe. But it still had mysterious religiousness. It was just different from the primitive and naive mysterious feeling of the swirling pattern of painted pottery. It possessed severe beauty and mystery by contrast. It was cloud scroll pattern that has inherited swirling patterns the most obvious. It was basically consisted of a helical element, and then made the element organized in a symmetry and repeated way. Cloud scroll pattern was the foundation of nephogram in Han dynasty. As regards the cloud patterns, there was not explicit appearance of cloud before Wei-and-Jin dynasties. Therefore, I discussed the mixture of cloud patterns and water patterns before Wei-and-Jin dynasties in this paper. As regards nephogram, the most remarkable feature was its long and dragging cloud tail which was similar to that of bent swirling pattern. We could find the transition relationship between the two patterns. However, to be apparent, nephogram was easier, freer and more insubstantial which got totally rid of the problem of liquid water. Such kind of painting made cloud pattern embodied with a state of gas. The primitive swirl pattern of painted pottery possessed a naive kind of mystery, and cloud thunder pattern in Shang-and-Zhou dynasties was ferocious and severe while the flowing cloud pattern in Qin-and-Han dynasties was vivid and insubstantial. All of them were produced by ancient people for their worship of primitive faith, which lead to rich mysterious and unpredictable feature of decorative patterns as a result.

The Period of Wei, Jin and Southern and Northern Dynasties was a unrest and highly religious times. It was meanwhile a times of national fusion. Taosim and Buddha developed equally while the arts from foreign culture were applied in the creation of patterns. Painting started to prevail and prompted the division of new categories, which also had influence on craft decoration. However, during the period when religion started to pervade, "the awareness of man" appeared. It was generated on the basis of the suspicion and negation of the slavery ideology with the original dominant position which was from classic books to predestination and from superstition to moral integrity." Such kind of "awareness" resulted in natural and adorable decoration with full flavor of life in this period. From the Period of Wei, Jin and Southern and Northern Dynasties to the Five Dynasties, the general appearance of decorative patterns has presented vivid and expressive temperament with harmonious and dynamic power. While after Wei-and-Jin dynasty, the focus on mystery has gradually been transferred to humanity. And religiousness began to fade down. Meanwhile, due to the effects from landscape painting, the depiction of water pattern tended to be natural in this period. The decorative grain type immersed formerly in mysterious state has turned to center on bright and clean natural images. The importance of humankind has been superior to divinity little by little, which has also affected the painting of water. And then the intimacy and harmonious between man and nature has been gradually reflected in the reappearance of water. It also showed its approachable character and attention on life and present.

After Tang and Song dynasties, the presentation of water pattern became more and more realistic and natural. It started to combine with many other images and some new propitious decorative patterns came into being, such as fish and dragon pattern, double dragons playing in water pattern, dragon and wave pattern and double carps playing in rippling, etc. Although water pattern in this period was not primary, as a background fill, it also played a significant and indispensable role on 
rendering the pattern atmosphere. Sometimes it was of great momentum, or of mild interest. Moreover, at this time, the mature of landscape painting caused the presentation of water patterns to be more specific and realistic. The development of craft arts made water of firmness and gentleness expressed everywhere in life through the bearing of various substances.

The marine traffic in Song dynasty has been developed completely. Therefore, many of navigation patterns appeared in arts and crafts. At this time, the development of water patterns have became prosperous accordingly. Besides the former water pattern was still in use continually, some new forms came into being in this dynasty. No matter as edging or major decoration, water patterns were applied much more widely at this time. Also, there were some enjoyable patterns with free forms of which the wave could be presented through several simple lines. Their forms were quite concise and the styles were sincere. It has been more meticulous and flexible for the conclusion of water patterns. For example, water patterns showed in "porcelain pillow with ducks playing in lotus pond", "porcelain cup with mandarin duck playing with lotus" and "lotus and water fowl patterns" have revealed fully artists' comfortable affection of nature and true feelings of life experiences. However, the creation of arts and crafts in Song dynasty was lack of magnificent vigor and great production. It was inclined to be graceful, elegant and implicit as well be full of literary tastes. The vigorous appearance of boldness and grandeur when in Tang dynasty has faded down gradually. The decorative patterns in Song dynasty has been free from religion and developed fast and realistically.

In Ming dynasty, the thoughts of arts and crafts were influenced by philosophy at that time when creating arts from pragmatic and practical angle was advocated. Thus, it was the peculiar styles of Ming dynasty that the patterns were honest and sincere with strong decorative functions. The same style was also reveled in water patters. From then on to Qing dynasty, the decorative pattern has followed the rules that the image must represent some certain meanings and the meanings must be propitious. The presentation of water patterns tended to represent good luck and happiness, such as Blessed Mountains and Longevity Sea, the harmonious four seas and the peaceful world, etc. Compared to that in Song dynasty, the style of decoration in Ming and Qing dynasties changed to be exquisite and complex. Water patterns headed for planar and produced stylized painting ways that a certain element was applied to repeat and overlap. The most typical pattern was the application of scaly water pattern. Though the water patterns in Ming and Qing dynasties were more abundant, the stylized painting ways have been formed and resulted in dull water patterns, and then developed towards pattering and systematism.

\section{Conclusions}

Chinese traditional decorative patterns bore the weight of Chinese traditional culture. It was the presentation of people's ideology and culture and aesthetic consciousness at that time as well the presentation of political economy in that society. Water pattern as one branch of Chinese traditional decorative patterns has developed constantly from the swirling patterns thousand years ago to the realistic patterns in contemporary times. In modern designs, the elements of water pattern usually appeared at the works of traditional styles. But in paintings and decorative books many of water patterns appeared as an image with few related document introductions. The same as other Chinese traditional decorative patterns, water patterns possessed fairly enormous and profound cultural conception. The conception of water was put forward by ancient people through their perception on natural laws and then lifted to their thoughts about the Ying-Yang, Five Elements and Doctrines. It has existed in Chinese traditional culture for thousand years and influenced the conception of many masters. Therefore, the origins and development of water pattern have been affected by the thoughts and cultural exchange in the society at that time. When we work at modern design, we should hold well the cultural connotation implied in the traditional decorative pattern and make a further understanding. Only if we paved the way for the inheritance of traditional decorative pattern, it could be supported by rich contents and progress further. 


\section{References}

1.Xu Xusheng. (2003). Legendary Period in Ancient China [M]. Guangxi Normal University Press, $10,130$.

2.Lin Haiyan. (2006). Visual Formation of Water Image---a Logical Form of Life [D]. Beijing: Central Academy of Fine Arts.

3.Xu Jing. (2004). A Study on the Multiple Culture of Water [J]. Journal of Hefei University of Technology (Social Sciences), 14, 65. 